\title{
MHD waves generated by high-frequency photospheric vortex motions
}

\author{
V. Fedun ${ }^{1}$, S. Shelyag ${ }^{2}$, G. Verth ${ }^{3,4}$, M. Mathioudakis ${ }^{2}$, and R. Erdélyi ${ }^{1}$ \\ ${ }^{1}$ Solar Physics \& Space Plasma Research Centre (SP2 RC), School of Mathematics and Statistics, University of Sheffield, \\ Hicks Building, Hounsfield Rd., Sheffield, S3 7RH, UK \\ ${ }^{2}$ Astrophysics Research Centre, School of Mathematics and Physics, Queen's University, Belfast, BT7 1NN, \\ Northern Ireland, UK \\ ${ }^{3}$ Centrum voor Plasma Astrofysica and Leuven Mathematical Modelling and Computational Science Centre, KU Leuven, \\ Celestijnenlaan 200B, 3001 Heverlee, Belgium \\ ${ }^{4}$ School of Computing, Engineering and Information Sciences, Northumbria University, Newcastle Upon Tyne, NE1 8ST, UK
}

Received: 27 January 2011 - Revised: 2 June 2011 - Accepted: 6 June 2011 - Published: 16 June 2011

\begin{abstract}
In this paper, we discuss simulations of MHD wave generation and propagation through a threedimensional open magnetic flux tube in the lower solar atmosphere. By using self-similar analytical solutions for modelling the magnetic field in Cartesian coordinate system, we have constructed a 3-D magnetohydrostatic configuration which is used as the initial condition for non-linear MHD wave simulations. For a driver we have implemented a highfrequency vortex-type motion at the footpoint region of the open magnetic flux tube. It is found that the implemented swirly source is able to excite different types of wave modes, i.e. sausage, kink and torsional Alfvén modes. Analysing these waves by magneto-seismology tools could provide insight into the magnetic structure of the lower solar atmosphere.
\end{abstract}

Keywords. Solar physics, astrophysics, and astronomy (Photosphere and chromosphere)

\section{Introduction}

There is observational evidence of a variety of magnetic field structures, i.e. open magnetic flux tubes, solar coronal loops, etc. in the higher layers of the solar atmosphere. These configurations, while in magnetohydrostatic equilibrium and thus relatively long-lived, are subject to wave motions, if a perturbation acts on them. The studies of recently reported observed ubiquitous wave motions in a range of mag-

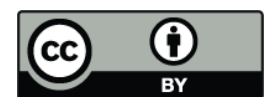

Correspondence to: V. Fedun (v.fedun@sheffield.ac.uk) netic field configurations in the photosphere, chromosphere and corona are of particular interest, since through different mechanisms of wave damping they might be responsible for the heating of the solar plasma (see e.g. Banerjee et al., 2007; Taroyan and Erdélyi, 2009). Furthermore, one can exploit these waves by the technique of solar magneto-seismology to probe the fine structure of the Sun's magnetised and highly dynamic atmosphere (see e.g. Goossens et al., 2002; Arregui et al., 2007; Erdélyi and Fedun, 2007; Goossens et al., 2008; Verth, 2008; Andries et al., 2005, 2009; Ruderman and Erdélyi, 2010). A popular excitation mechanism of waves in magnetic chromospheric and coronal structures is the leakage of oscillatory modes from the inner layers of the solar photosphere along magnetic regions, because the magnetic field favourably shifts the acoustic cut-off frequency.

Periodic motions at the footpoint regions of magnetic flux tubes can generate waves that may supply energy to the upper part of the solar atmosphere. For example, granular buffeting motion could be responsible for the excitation of kink (transverse) waves. Turbulence in the convection zone near the surface of the Sun excites solar p-modes (Goldreich and Keeley, 1977). These acoustic waves are transmitted to the photospheric region of the solar atmosphere and are able to drive longitudinal magneto-acoustic waves in magnetic flux tubes. These motions have large spatial scales. A variety of one- and multi-dimensional MHD numerical simulations of wave generation in a solar magnetic flux tube by various periodic vertical or horizontal sources have already been performed (see for example Bogdan et al., 2003; Hasan et al., 2005; Vigeesh et al., 2009; Ofman, 2009; Fedun et al., 2009; Felipe et al., 2010; Fedun et al., 2011). Here we push these frontiers further. 


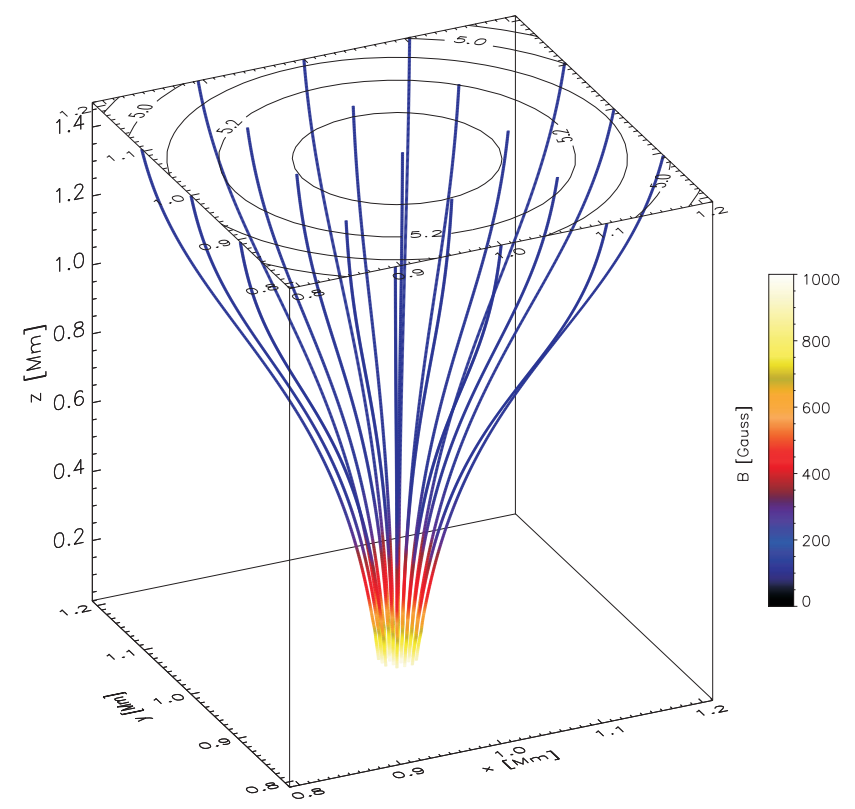

Fig. 1. Initial magnetic flux tube configuration. Selected colored lines correspond to the magnetic field lines. Also, color indicates the value of the magnetic field strength at different regions of the flux tube. The iso-contours of the initially constant magnetic field are overplotted at the top of the simulation box. Note, only central part (i.e. $x, y=[0.8,1.2] \mathrm{Mm}$ ) of the full computational domain (i.e. $x, y=[0,2] \mathrm{Mm})$ is shown.

Modern state-of-the-art ground- and space-based solar observational instruments e.g. Swedish Solar Telescope (SST), DST/ROSA, Hinode, SDO etc., carry out the highest spatiotemporal resolution of dynamical processes in the lower and upper regions of the solar atmosphere (see e.g. Bonet et al., 2008; Wedemeyer-Böhm and Rouppe van der Voort, 2009; Wedemeyer-Böhm, 2010).

Recently, Bonet et al. (2008) have discovered localised vortex-type motions created at downdrafts where the plasma returns to the solar interior after cooling down. The reported swirls have diameters $0.2-1.5 \mathrm{Mm}$ and lifetimes of 3-7 min. They have found numerous examples of convectively driven vortex flows with clockwise and counterclockwise rotation. Wedemeyer-Böhm and Rouppe van der Voort (2009) have analysed the time series of spectral scans through the Ca II $854.2 \mathrm{~nm}$ spectral line with the CRISP instrument mounted at the Swedish Solar Telescope and found rotations up in the chromosphere. They interpret these swirly motions and the associated bright point motions as a direct indication of upper-atmospheric magnetic field twisting and braiding as a result of convective buffeting of magnetic footpoints. We show that such observed rotational motions could be a natural driver for not just Alfvén but a range of MHD waves, i.e., slow (SMAW) and fast (FMAW) magnetoacoustic waves.

Previously, the torsional Alfvén wave has been studied theoretically by (e.g. Hollweg, 1981; Hollweg et al., 1982).
In these papers the authors have analysed the linearised propagation of axisymmetric twists on axisymmetric vertical flux tubes. Open and closed configurations of the magnetic flux tubes which may model e.g. the coronal hole and active region loops have been studied. It was found that torsional Alfvén waves may produce enough energy flux to heat the solar corona (see also Antolin et al., 2008).

Verth et al. (2010) have shown that observation of the eigenmodes of torsional Alfvén waves can provide temperature diagnostics of both the internal and surrounding plasma, i.e. these waves are the ideal magneto-seismological tool for probing radial plasma inhomogeneity in solar waveguides.

Due to the incompressible nature of the Alfvén wave the detection of this type of wave MHD mode is rather challenging and is difficult in the solar atmosphere. Only recently Jess et al. (2009), by studying of a chromospheric magnetic bright point (MBP) group and analysing periodic $\mathrm{H} \alpha$ spectral line broadening with no intensity variations, have clearly shown the existence of this wave mode in the Sun.

Numerically, vortex-type motion in the radiative MHD simulations of magnetoconvection has recently been found by Shelyag et al. (2011a,b). These authors have shown a direct connection between magnetic vortices and rotary motions of photospheric bright points, and suggested that there may be a connection between the MBP rotation and smallscale swirly motions observed higher in the atmosphere.

Based on these theoretical, observational and numerical results, in this short paper, we focus on waves emanating from a spatially localised vortex source at the footpoint region of an open magnetic flux tube. Due to the swirly motion it is anticipated that such drivers are the natural exciters of torsional Alfvén waves. Analysing the evolution of the horizontal cross section of a simulated magnetic flux tube we are able to establish clear evidence of propagating MHD kink and sausage modes as well.

\section{Numerical model and simulations}

We use the 3-D version of Sheffield Advanced Code (SAC) to perform our simulations (for more details about code see Shelyag et al., 2008). The computational domain has a physical size of $2 \times 2 \mathrm{Mm}$ in the horizontal directions $x$ and $y$, and in the vertical direction $z$ the domain extends from the lower photosphere through the chromosphere up to the transition region i.e. $1.6 \mathrm{Mm}$ in height. Numerically the domain is resolved by $100 \times 100 \times 196$ grid cells in the $\mathrm{x}-$, $\mathrm{y}-$ and $\mathrm{z}$ directions, respectively. The boundaries of the computational box are set to be open. The standard VAL IIIC (Vernazza et al., 1981) model profiles of temperature and density have been used as a realistic stratified hydrostatic background.

The self-similar approach is used to construct the nonpotential 3-D magnetic field configuration, i.e., open magnetic flux tube. Schlüter and Temesváry (1958) were the first who suggested to use self-similar magnetic field 

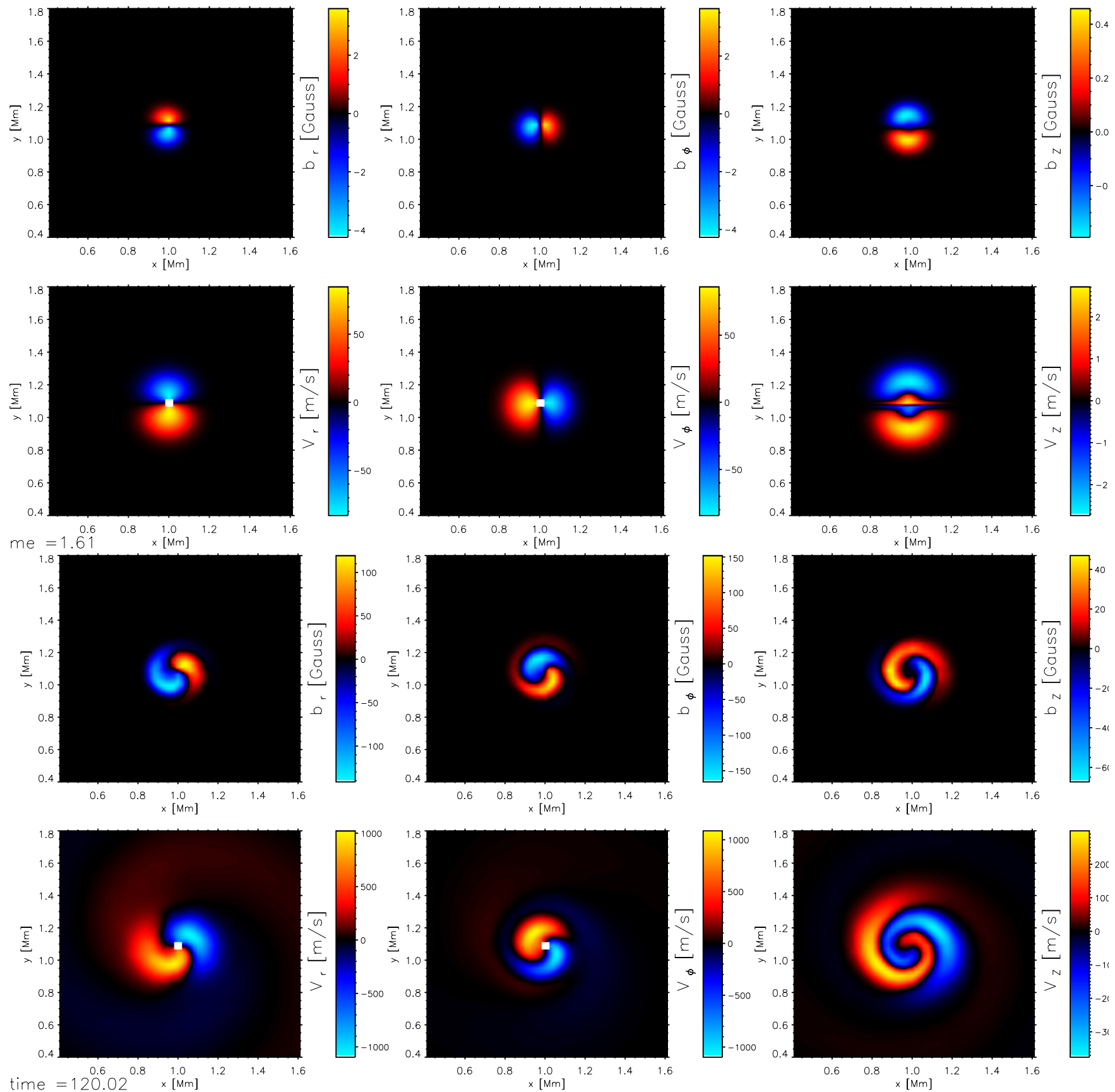

Fig. 2. Rendering of the magnetic field $\left(b_{\mathrm{r}}, b_{\phi}, b_{\mathrm{Z}}\right)$ and velocity $\left(V_{\mathrm{r}}, V_{\phi}, V_{\mathrm{z}}\right)$ perturbation components generated by the vortex-type periodic driver (see Eq. 2) at the footpoint of the magnetic flux tube. Two different times are shown. The top and bottom sets of six horizontal slices at hight $h=0.12 \mathrm{Mm}$ correspond to simulation times $t=1.5 \mathrm{~s}$ and $t=120.02 \mathrm{~s}$, respectively.

constructions for description of various solar magnetic phenomena. Currently, magnetic fields of this type are widely used in numerical simulations in helioseismology and coronal physics (e.g. Gordovskyy and Jain, 2007; Cameron et al., 2008; Shelyag et al., 2009; Fedun et al., 2011). In our particular three-dimensional case, due to the self-similarity, the open magnetic flux tube is obtained analytically from the following set of equations:

$$
\begin{aligned}
& B_{\mathrm{x}}=-\frac{x}{2} \frac{d B_{0 z}(z)}{d z} \cdot G(f), \\
& B_{\mathrm{y}}=-\frac{y}{2} \frac{d B_{0 z}(z)}{d z} \cdot G(f), \\
& B_{\mathrm{z}}=B_{0 z(z)} \cdot G(f),
\end{aligned}
$$



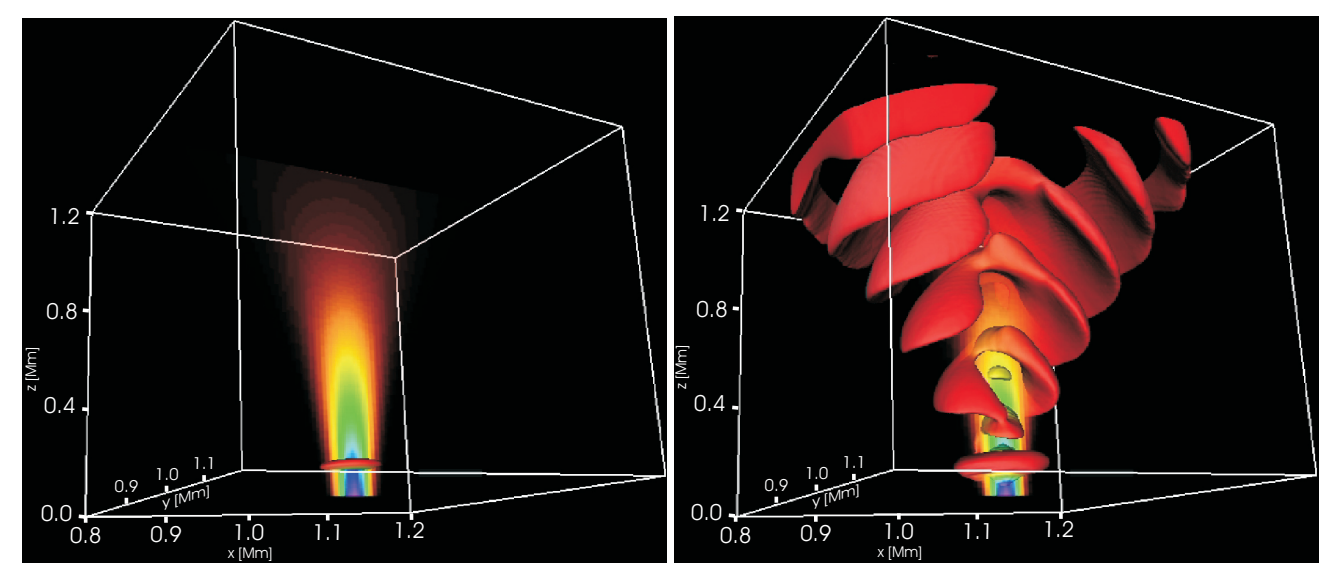

Fig. 3. Snapshots showing the iso-surfaces of the horizontal velocity component $V_{\mathrm{X}}$ during numerical simulation. From left to the right the snapshots are at $t=7.2,312 \mathrm{~s}$ at the centre of the numerical domain we have overplotted the 2-D vertical slice of the background $B_{\mathrm{Z}}$ component of the magnetic field. Note, rendering of the 3-D numerical data has been constructed using the VAPOR visualisation package.
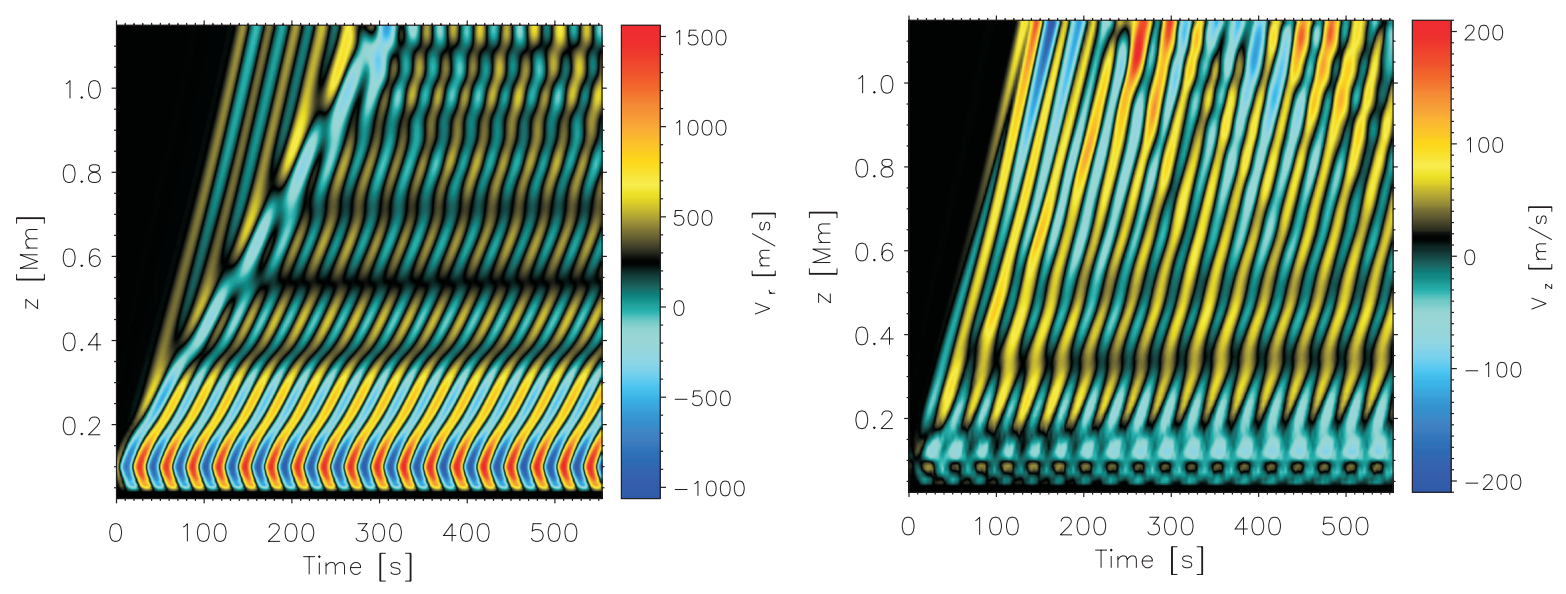

Fig. 4. The time-distance diagrams of the radial $\left(V_{\mathrm{r}}\right)$ and the vertical $\left(V_{\mathrm{Z}}\right)$ components of the velocity at $x=0.98 \mathrm{Mm}$ and $y=0.98 \mathrm{Mm}$, i.e., near the axis of the magnetic flux tube.

and

$f=\frac{x^{2}+y^{2}}{r_{0}^{2}} \cdot B_{0 z}(z)$

where $B_{0 z}(z)$ is the vertical $z$ component of the background magnetic field along the symmetry axis towards the top boundary of the model, $r_{0}$ is a radial scaling and $G$ is an arbitrarily chosen function which describes the profile in the radial direction of the vertical magnetic field component. In the present case this function is chosen to be exponential, i.e.

$G(f)=A \cdot \exp (-f)$,

where $A$ is an arbitrary constant. To implement this magnetic field configuration into the background hydrostatic equilibrium, we have used the same principles as Fedun et al. (2011) for a two-dimensional case. The constructed 3-D magnetic flux tube is then visualised by selected magnetic field lines as demonstrated in Fig. 1. The different colors along the field lines correspond to magnetic field strength. The magnetic field strength at the footpoint region is $B_{0}=1000 \mathrm{G}$ and reduces with height in the mid-chromosphere to a few $\mathrm{G}$ (in the horizontal direction the magnetic field has a maximum absolute value at the centre and minimum value at edges of the flux tube). The footpoint flux tube radius is $100 \mathrm{~km}$.

In the Introduction we have noted that vortex-type motion at the photospheric region is widely observed. Swirly motions and associated MBP motions are a direct indication of upper-atmospheric magnetic field twisting and braiding as a result of convective buffeting of magnetic footpoints. Based on these observational results we have implemented here a vortex-type high-frequency periodic source located at the footpoint of the magnetic flux tube as an initial driver in our numerical simulations. The $V_{\mathrm{x}}$ and $V_{\mathrm{y}}$ components of the velocity perturbation have a Gaussian spatial distribution in the $\mathrm{x}-, \mathrm{y}$ - and $\mathrm{z}$-directions: 

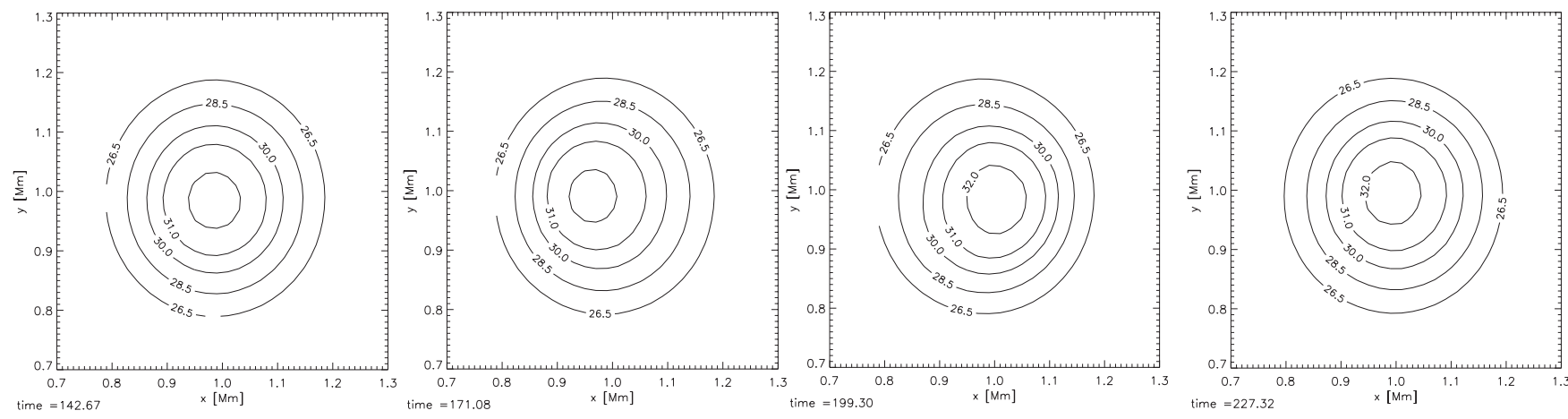

Fig. 5. A time series of horizontal cuts at height $h=0.75 \mathrm{Mm}$ of the total magnetic field. The strength of the magnetic field is shown as iso-contours. The difference in time between the snapshots is approximately equal to $30 \mathrm{~s}$, i.e., the period of footpoint driver. Note the clear evidence of the sausage and kink oscillations which propagate along the magnetic flux tube.

$V_{\mathrm{x}}=A_{0} e^{-\frac{\left(r-r_{0}\right)^{2}}{\Delta r^{2}}} e^{-\frac{\left(z-z_{0}\right)^{2}}{\Delta z^{2}}} \sin \left(\frac{2 \pi t}{T}\right)$,
$V_{\mathrm{y}}=A_{0} e^{-\frac{\left(r-r_{0}\right)^{2}}{\Delta r^{2}}} e^{-\frac{\left(z-z_{0}\right)^{2}}{\Delta z^{2}}} \cos \left(\frac{2 \pi t}{T}\right)$,

where $A_{0}$ is the amplitude of the initial perturbation; $\Delta r$ and $\Delta z$ are the half width of the Gaussian spatial profiles of the driver in the radial and vertical directions, respectively; $T$ is the period of the driver, $r^{2}=x^{2}+y^{2}$ is the radial distance. In our numerical simulations $\Delta r=0.1 \mathrm{Mm}$ and $\Delta z=0.01 \mathrm{Mm}$ in order to mimic observed drivers.

In Fig. 2 the initial state and disturbances generated by a vortex driver at a later elapsed time $(t=120 \mathrm{~s})$ are shown as a two-dimensional horizontal slice at height $h=0.12 \mathrm{Mm}$, i.e. just above the region where the driver is located in the computational domain. We have plotted a color rendering of the perturbed components of the magnetic field $\left(b_{\mathrm{r}}, b_{\phi}\right.$, $\left.b_{\mathrm{z}}\right)$ and velocities $\left(V_{\mathrm{r}}, V_{\phi}, V_{\mathrm{z}}\right)$ in cylindrical coordinate system. Disturbances excited by the implemented vortex driver in the computational domain are similar to those obtained by Shelyag et al. (2011a,b) in direct radiative MHD simulations of magneto-convection and observed vortex motions by Bonet et al. (2008) in photospheric G-band bright points.

The horizontal component of velocity $V_{\mathrm{x}}$ is shown in Fig. 3 as isosurfaces. Illustrative snapshots are captured at times $t=7.2$ and $312 \mathrm{~s}$, respectively. At the centre of each snapshot we have overplotted the vertical cut of the background magnetic field component $B_{\mathrm{Z}}$ which shows the internal magnetic structure of the constructed magnetic flux tube. The high frequency $(T=30 \mathrm{~s})$ vortex driver is located at the footpoint region of the magnetic flux tube (see the first upper left panel of Fig. 3).

After ten periods (see the right panel of Fig. 3) we observe a 3-D pattern of the SMAW and FMAW distribution in the computational domain. The SMAWs are located near the axis of the magnetic flux tube and propagate vertically upward, while the FMAWs propagate obliquely. It can be seen that SMAWs, generated by the driver, are weak and present only up to the height $h=0.7 \mathrm{Mm}$, i.e., SMAWs do not supply considerable energy to the upper part of the chromosphere. Note, in a 3-D geometry we can now clearly see the shape of generated waves. SMAWs have a maximum of amplitude at the axis of the magnetic flux tube, equal to $800 \mathrm{~m} \mathrm{~s}^{-1}$.

Time-distance diagrams of the radial $\left(V_{\mathrm{r}}\right)$ and vertical $\left(V_{\mathrm{z}}\right)$ components of the velocity rendered approximately at the axis of the magnetic flux tube are shown in Fig. 4. As we have noted previously, two types of waves with different phase speeds can be resolved. The SMAW and FMAW propagate with phase speeds $V_{\mathrm{ph}}=3.8 \mathrm{~km} \mathrm{~s}^{-1}$ and $V_{\mathrm{ph}}=$ $8.2 \mathrm{~km} \mathrm{~s}^{-1}$, respectively.

The dark horizontal regions on the time-distance diagram of the radial component $\left(V_{\mathrm{r}}\right)$ (see left panel of Fig. 4) occur due to wave interference (both SMAW and FMAW) in the radial direction.

In Fig. 5 we have shown iso-contours of the total magnetic field at $h=0.75 \mathrm{Mm}$ for simulation times $t \approx 142,171$, 199 and 227. It is clearly seen that the area inside, e.g., the iso-contour at 32 Gauss, decreases and increases periodically. This is clear evidence of a sausage mode excited by a vortex driver. Could this be observed? Our prediction of generated sausage waves has recently been confirmed observationally by Morton et al. (2011). Furthermore, this driver also excites the kink mode as is shown by transverse motion of the magnetic flux tube centre (see Fig. 5). We appreciate that is difficult to identify the changes of position and areas of iso-contours in static images. To help readers, we also provided movies in downloadable electronic form, that can be found at http://swat.group.shef.ac.uk/simulations.html.

\section{Conclusions}

The purpose of this short report is to investigate numerically the generation of MHD waves in an open magnetic flux tube by a vortex-type photospheric driver in a very realistic 
three-dimensional geometry. We have shown that a such driver can excite both SMAW and FMAW. The torsional Alfvén wave is also present and the interesting properties of this mode will be a focus of our future study. There is simply no space available here for a deep analysis. We have not investigated the possible heating contribution of the generated MHD modes. This requires much more detailed numerical and analytical analysis. For more efficient heating due to MHD waves, these waves must transfer their energy to smaller length scales due to various types of instability and dissipation mechanisms (see Fedun et al., 2004; Copil et al., 2010). In order to resolve the dissipation numerically very powerful high performance computational equipment is needed and we hope to make the first steps in this direction in the near future.

Acknowledgements. The authors thank The Royal Society (UK) for the financial support they received. RE acknowledges M. Kéray for patient encouragement and is grateful to NSF, Hungary (OTKA, Ref. No. K83133). G.V. acknowledges financial support from Northumbria University and K.U.Leuven via GOA/2009-009.

Guest Editor M. Balikhin thanks two anonymous referees for their help in evaluating this paper.

\section{References}

Andries, J., Arregui, I., and Goossens, M.: Determination of the Coronal Density Stratification from the Observation of Harmonic Coronal Loop Oscillations, ApJL, 624, L57-L60, 2005.

Andries, J., van Doorsselaere, T., Roberts, B., Verth, G., Verwichte, E., and Erdélyi, R.: Coronal Seismology by Means of Kink Oscillation Overtones, Space Sci. Rev., 149, 3-29, 2009.

Antolin, P., Shibata, K., Kudoh, T., Shiota, D., and Brooks, D.: Predicting Observational Signatures of Coronal Heating by Alfvén Waves and Nanoflares, ApJ, 688, 669-682, 2008.

Arregui, I., Andries, J., Van Doorsselaere, T., Goossens, M., and Poedts, S.: MHD seismology of coronal loops using the period and damping of quasi-mode kink oscillations, A\&A, 463, 333338, 2007.

Banerjee, D., Erdélyi, R., Oliver, R., and O'Shea, E.: Present and Future Observing Trends in Atmospheric Magnetoseismology, SolPhys, 246, 3-29, 2007.

Bogdan, T. J., Carlsson, M., Hansteen, V. H., McMurry, A., Rosenthal, C. S., Johnson, M., Petty-Powell, S., Zita, E. J., Stein, R. F., McIntosh, S. W., and Nordlund, Å.: Waves in the Magnetized Solar Atmosphere. II. Waves from Localized Sources in Magnetic Flux Concentrations, ApJ, 599, 626-660, 2003.

Bonet, J. A., Márquez, I., Sánchez Almeida, J., Cabello, I., and Domingo, V.: Convectively Driven Vortex Flows in the Sun, ApJ, 687, L131-L134, 2008.

Cameron, R., Gizon, L., and Duvall Jr., T. L.: Helioseismology of Sunspots: Confronting Observations with Three-Dimensional MHD Simulations of Wave Propagation, Solar Phys., 251, 291308, 2008.

Copil, P., Voitenko, Y., and Goossens, M.: Torsional Alfvén waves in small scale current threads of the solar corona, A\&A, 510, A17-+, 2010.
Erdélyi, R. and Fedun, V.: Are There Alfvén Waves in the Solar Atmosphere?, Science, 318, 1572-1574, 2007.

Fedun, V. N., Yukhimuk, A. K., and Voitsekhovskaya, A. D.: The transformation of MHD Alfvén waves in space plasma, J. Plasma Phys., 70, 699-707, 2004.

Fedun, V., Erdélyi, R., and Shelyag, S.: Oscillatory Response of the 3D Solar Atmosphere to the Leakage of Photospheric Motion, SolPhys, 258, 219-241, 2009.

Fedun, V., Shelyag, S., and Erdélyi, R.: Numerical Modeling of Footpoint-driven Magneto-acoustic Wave Propagation in a Localized Solar Flux Tube, ApJ, 727, 17-31, 2011.

Felipe, T., Khomenko, E., and Collados, M.: Magneto-acoustic Waves in Sunspots: First Results From a New Three-dimensional Nonlinear Magnetohydrodynamic Code, ApJ, 719, 357-377, 2010.

Goldreich, P. and Keeley, D. A.: Solar seismology. II - The stochastic excitation of the solar p-modes by turbulent convection, ApJ, 212, 243-251, 1977.

Goossens, M., Andries, J., and Aschwanden, M. J.: Coronal loop oscillations. An interpretation in terms of resonant absorption of quasi-mode kink oscillations, A\&A, 394, L39-L42, 2002.

Goossens, M., Arregui, I., Ballester, J. L., and Wang, T. J.: Analytic approximate seismology of transversely oscillating coronal loops, A\&A, 484, 851-857, 2008.

Gordovskyy, M. and Jain, R.: Scattering of p-Modes by a Thin Magnetic Flux Tube, ApJ, 661, 586-592, 2007.

Hasan, S. S., van Ballegooijen, A. A., Kalkofen, W., and Steiner, O.: Dynamics of the Solar Magnetic Network: Two-dimensional MHD Simulations, ApJ, 631, 1270-1280, 2005.

Hollweg, J. V.: Alfven waves in the solar atmosphere. II - Open and closed magnetic flux tubes, SolPhys, 70, 25-66, 1981.

Hollweg, J. V., Jackson, S., and Galloway, D.: Alfven waves in the solar atmosphere. III - Nonlinear waves on open flux tubes, SolPhys, 75, 35-61, 1982.

Jess, D. B., Mathioudakis, M., Erdélyi, R., Crockett, P. J., Keenan, F. P., and Christian, D. J.: Alfvén Waves in the Lower Solar Atmosphere, Science, 323, 1582-1585, 2009.

Morton R., Erdélyi, R., Jess D., and Mathioudakis, M.: Observations of sausage modes in magnetic pores, ApJ Letters, 729, L18-L21, 2011.

Ofman, L.: Progress, Challenges, and Perspectives of the 3D MHD Numerical Modeling of Oscillations in the Solar Corona, Space Sci. Rev., 149, 153-174, 2009.

Ruderman, M. S. and Erdélyi, R.: Transverse Oscillations of Coronal Loops, Space Sci. Rev., 149, 199-228, 2010.

Schlüter, A. and Temesváry, S.: The Internal Constitution of Sunspots, Electromagnetic Phenomena in Cosmical Physics, 6, 263-274, 1958.

Shelyag, S., Fedun, V., and Erdélyi, R.: Magnetohydrodynamic code for gravitationally-stratified media, A\&A, 486, 655-662, 2008.

Shelyag, S., Zharkov, S., Fedun, V., Erdélyi, R., and Thompson, M. J.: Acoustic wave propagation in the solar sub-photosphere with localised magnetic field concentration: effect of magnetic tension, A\&A, 501, 735-743, 2009.

Shelyag, S., Keys, P., Mathioudakis, M., and Keenan, F. P.: Vorticity in the solar photosphere, A\&A, 526, A5-, 2011.

Shelyag, S., Fedun, V., Keenan, F. P., Erdélyi, R., and Mathioudakis, M.: Photospheric magnetic vortex structures, Ann. Geophys., 29, 
883-887, doi:10.5194/angeo-29-883-2011, 2011.

Taroyan, Y. and Erdélyi, R.: Heating Diagnostics with MHD Waves, Space Sci. Rev., 149, 229-254, 2009.

Vernazza, J. E., Avrett, E. H., and Loeser, R.: Structure of the solar chromosphere. III - Models of the EUV brightness components of the quiet-sun, ApJ Supplement Series, 45, 635-725, 1981.

Verth, G.: Magneto-seismology: effect of inhomogeneous magnetic field on transversal coronal loop oscillations, edited by: Erdélyi, R. and Mendoza-Bricẽno, C. A., IAU Symp., (Cambridge: Cambridge Univ. Press), 247, 123-132, 2008.

Verth, G., Erdélyi, R., and Goossens, M.: Magnetoseismology: Eigenmodes of Torsional Alfvén Waves in Stratified Solar Waveguides, ApJ, 714, 1637-1648, 2010.
Vigeesh, G., Hasan, S. S., and Steiner, O.: Wave propagation and energy transport in the magnetic network of the Sun, A\&A, 508, 951-962, 2009.

Wedemeyer-Böhm, S.: Small-scale structure and dynamics of the chromospheric magnetic field, MmSAI, 81, 693-700, 2010.

Wedemeyer-Böhm, S. and Rouppe van der Voort, L.: Small-scale swirl events in the quiet Sun chromosphere, A\&A, 507, L9-L12, 2009. 